\title{
ACCURACY ASSESSMENT OF CROWN DELINEATION METHODS FOR THE INDIVIDUAL TREES USING LIDAR DATA
}

\author{
K. T. Chang a, *, C. Lin' ${ }^{\mathrm{b}}$ Y. C. $\operatorname{Lin}^{\mathrm{c}}$, J. K. Liu ${ }^{\mathrm{d}}$ \\ a. Associate Professor, Dept. of Civil Eng. and Environmental Informatics, Ming Hsin University of Science and Technology, \\ Hsinchu County 30401, Taiwan, ktchang1216@gmail.com \\ b. Professor, Dept. of Forestry and Natural Resources, National Chiayi University, Chiayi, Taiwan, chinsu@mail.ncyu.edu.tw \\ c. Assistant Professor, Dept. of Environmental Information and Engineering, Chung Cheng Institute of Technology, \\ National Defense University, Taoyuan County 30401, Taiwan, yuching.ncl@ gmail.com \\ d. CEO, LIDAR Technology Co., Ltd., Hsinchu County 30274, Taiwan, jkliu@lidar.com.tw
}

\section{Commission VIII, WG VIII/7}

\begin{abstract}
Forest canopy density and height are used as variables in a number of environmental applications, including the estimation of biomass, forest extent and condition, and biodiversity. The airborne Light Detection and Ranging (LiDAR) is very useful to estimate forest canopy parameters according to the generated canopy height models (CHMs). The purpose of this work is to introduce an algorithm to delineate crown parameters, e.g. tree height and crown radii based on the generated rasterized CHMs. And accuracy assessment for the extraction of volumetric parameters of a single tree is also performed via manual measurement using corresponding aerial photo pairs. A LiDAR dataset of a golf course acquired by Leica ALS70-HP is used in this study. Two algorithms, i.e. a traditional one with the subtraction of a digital elevation model (DEM) from a digital surface model (DSM), and a pit-free approach are conducted to generate the CHMs firstly. Then two algorithms, a multilevel morphological active-contour (MMAC) and a variable window filter (VWF), are implemented and used in this study for individual tree delineation. Finally, experimental results of two automatic estimation methods for individual trees can be evaluated with manually measured stand-level parameters, i.e. tree height and crown diameter. The resulting CHM generated by a simple subtraction is full of empty pixels (called "pits") that will give vital impact on subsequent analysis for individual tree delineation. The experimental results indicated that if more individual trees can be extracted, tree crown shape will became more completely in the CHM data after the pit-free process.
\end{abstract}

KEY WORDS: Forest, Canopy, LiDAR, Pit-free, Stand-level

\section{INTRODUCTION}

A LiDAR system comprises multiple subsystems, namely a global positioning system (GPS), inertial measurement unit, and laser scanner. Currently, the large volumes of spatial data collected in a short period using LiDAR systems are typically employed for quantitative analyses and modeling in studies on geology, coastal erosion, and geomorphology. Airborne LiDAR technology can be used to collect multiple laser returns at pulse repetition rates up to $500 \mathrm{KHz}$. The positional accuracy of the resultant laser pulse return is typical at the decimeter level. Thus, the obtained standard products of an airborne LiDAR survey included all points, ground points, digital surface models (DSM), and digital elevation models (DEMs). Two types of airborne LiDAR systems are currently available; full-waveform (FW) and discrete-echo LiDAR. Regarding discrete-echo LiDAR systems, the return signal is filtered to export multiple echoes. For each transmitted laser pulse, only three to seven echoes are typically used to record the intensity and three-dimensional coordinates. However, FW LiDAR systems can record the entire waveform for each transmitted laser pulse. A waveform typically comprises responses per nanosecond. Therefore, a maximum of 255 echoes can be obtained. Thus, an unprecedented level of information can be preserved. Additional land information and high density point cloud data can be acquired, improving the accuracy of digital terrain models (DTMs). Because the reflected waveforms are affected by the type of material and properties of detected land objects, the waveform features can also be employed to analyze the characteristics associated with land cover change. LiDAR systems have been applied in close-range or aerial topographical surveys, to classify vegetation in forested areas, and map disasters (Wehr \& Lohr, 1999). To estimate the forest canopy structures or individual crown structure by LIDAR technology, it is a very good method (Lin et al., 2011; Lo \& Lin, 2013). For the single standing-based approach, canopy parameters of individual trees can be extracted from the Canopy Height Models (CHMs). Except for the tree height, there are some parameters such as crown width (CW), crown base height, and crown projected area (CPA) also can be estimated in the process (Bortolot \& Wynne, 2005; Popescu, 2007; Kwak et al., 2010; Dalponte et al., 2011).

The purpose of this work is to perform a comparison of different CHMs generation methods for subsequent processes of the extraction of volumetric parameters of a single tree either directly or indirectly. Two algorithms, i. e. a subtraction of digital surface model (DSM) and digital elevation model (DEM), and a pit-free approach

\footnotetext{
* Corresponding author
} 
are conducted to generate the CHMs firstly. Then a multilevel morphological active-contour (MMAC) algorithm was used for individual tree delineation. Finally, the rasterized CHMs derived from two algorithms were compared with measured stand-level parameters, i.e. tree height and crown width $(\mathrm{CW})$.

\section{METHODOLOGY}

\subsection{CHM Generation}

The most simple CHM generation method is a subtraction of digital surface model (DSM) and digital elevation model (DEM). However, there are some improvement for the CHM generation, e.g. searching the highest LiDAR return in each grid cell, replacing each LiDAR return with a small disk, using TIN interpolation, and a pit-free method developed by Khosravipour et al. (2014).

A simple improvement can be obtained by replacing each LiDAR return with a small disk. After all, depending on the flying height the laser beam has a diameter of 10 to 50 centimeter and approximating this with a single point of zero area seems overly conservative. But this produces increasingly smooth CHMs with widening tree crowns. This "splats" the LiDAR returns into circles that are growing larger and larger than the laser beam diameter and thus have less and less in common with reality.

Another popular approach avoids this by interpolating all first returns with a triangulated irregular network (TIN) and then rasterizing it onto a grid to create the CHM. The result has no more empty pixels but is full of pits because many laser pulses manage to deeply penetrate the canopy before producing the first return. When combining multiple flight lines some laser pulses may have an unobstructed view of the ground under the canopy without hitting any branches. These "pits" and how to avoid them is discussed in great length in the September 2014 edition of the ASPRS PE\&RS journal by a paper of Khosravipour et al. A LAStools developed by the Rapidlasso $\mathrm{GmbH}$ had implemented these ideas as the following. First, combining the '-highest' gridding and TIN interpolation. Next, the idea of expanding the points into circles with a diameter of 5 centimeter to account for the laser beam diameter by adding option "-subcircle 0.025 " to "lasthin" function in the LAStools (Isenburg, 2014).

\subsection{MMAC Algorithm}

The MMAC algorithm combines a multi-level morphological approach with the active contour model described previously. The algorithm proposed in this study is based on mathematical morphology (MM) concepts and uses an adapted watershed segmentation technique The MMAC algorithm is comprised of three steps. The first step uses bottom up erosion (BUE) to process CHM data and locate the stand candidates within a forested area. In the MMAC algorithm, stand candidates are identified by an iterative process in which image data is successively eroded from the bottom up until finally the highest points are determined. The second step uses a top down dilation (TDD) technique to estimate tree crown periphery points by growing outwards from stand candidate central points. The third step uses an active contour model (ACM) to modify the periphery points and delineate the contours of the tree crown boundary (Lin et al., 2011; Lo \& Lin, 2013).

\section{CASE STUDY}

\subsection{Study area}

The test area located at a Golf course in the Hsinchu county. A Leica ALS 70-HP scanner was used in the experiment. The scanning date is Oct. 17, 2013, the flight altitude is $1295 \mathrm{~m}$, pulse rate is $210 \mathrm{KHz}$, and point density is $2.483 \mathrm{pt} / \mathrm{m}^{2}$. The acquired point cloud covered the study area is shown as Figure 1 . Two test area $(300 \mathrm{~m}$ $\mathrm{x} 300 \mathrm{~m}$ ) are clipped from the original point cloud for the subsequently analysis. The clipped point clouds for the test area \#1 and \#2 are also shown as right parts in the Figure 2.

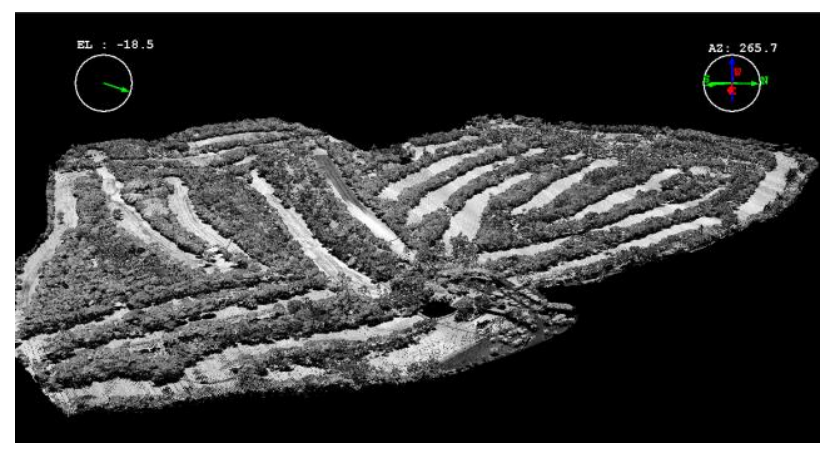

Figure 1. The point cloud covering the study area

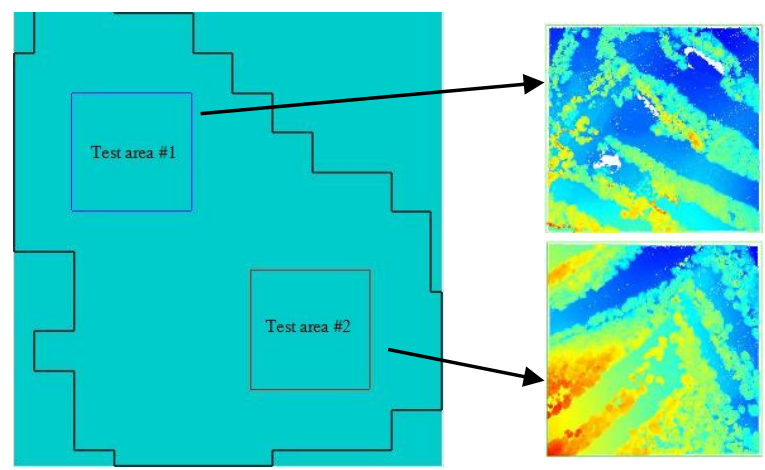

Figure 2. Two test areas in the golf course area bounded by the black line.

To test the accuracy of different individual crown delineation (ICD) methods, stereo measurement in the aerial image pairs is used to derive the volumetric parameters of 27 trees as a reference data in the test area $\# 1$, as shown in Figure 3. In the figure, red points represent horizontal position for the treetop of 27 trees. 


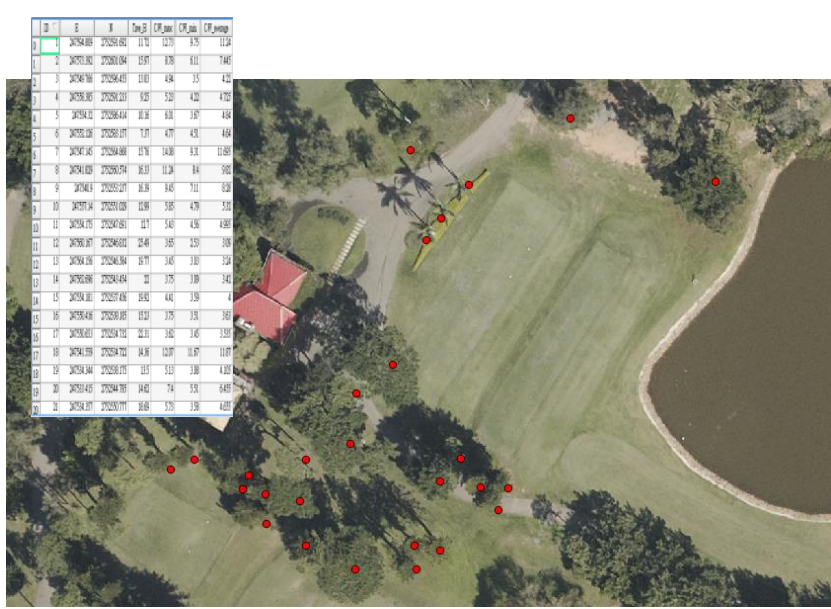

Figure 3. Reference data for the accuracy assessment of ICD methods

\subsection{Test results}

3.2.1 Results for the early study: A TreeTop application developed in a Web-LiDAR forest inventory project was conducted to perform individual tree delineation for the CHMs comparison (Chang et al., 2015). Web-LiDAR was developed to support LiDARbased forest inventory and management at Eglin Air Force Base (AFB), Florida, USA (Silva et al., 2014). The ICD algorithm called VWF is performed using a threshold to find tree tops and using a variable window to describe the tree crown width (TCW) is a function of tree height (Popscu \& Wynne, 2004). The individual tree detection results for the simple subtraction CHM and one for the pit-free CHM are shown as Figures 4 and 5, respectively. It shows too many duplicated tree tops as shown in the figures.

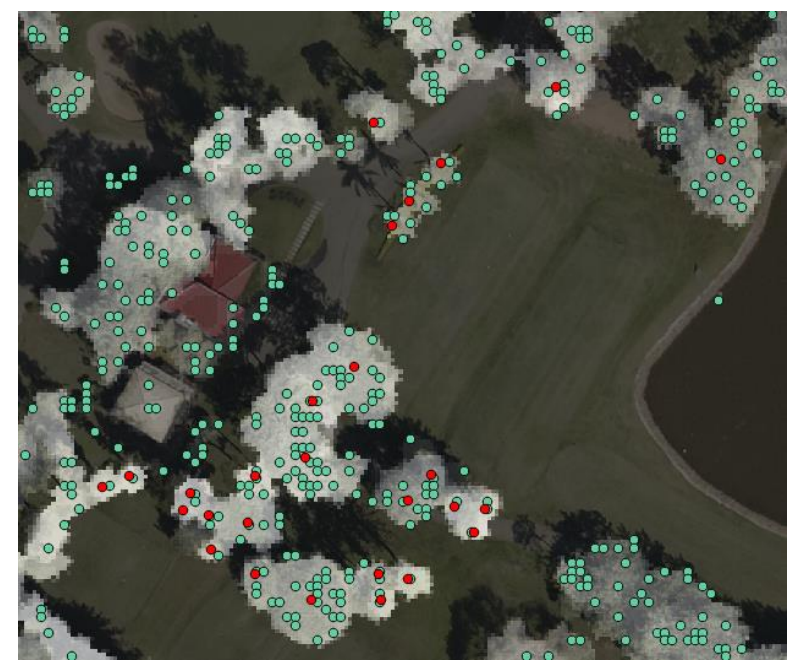

Figure 4. Extraction result of the VWF method using simple subtraction CHMs.

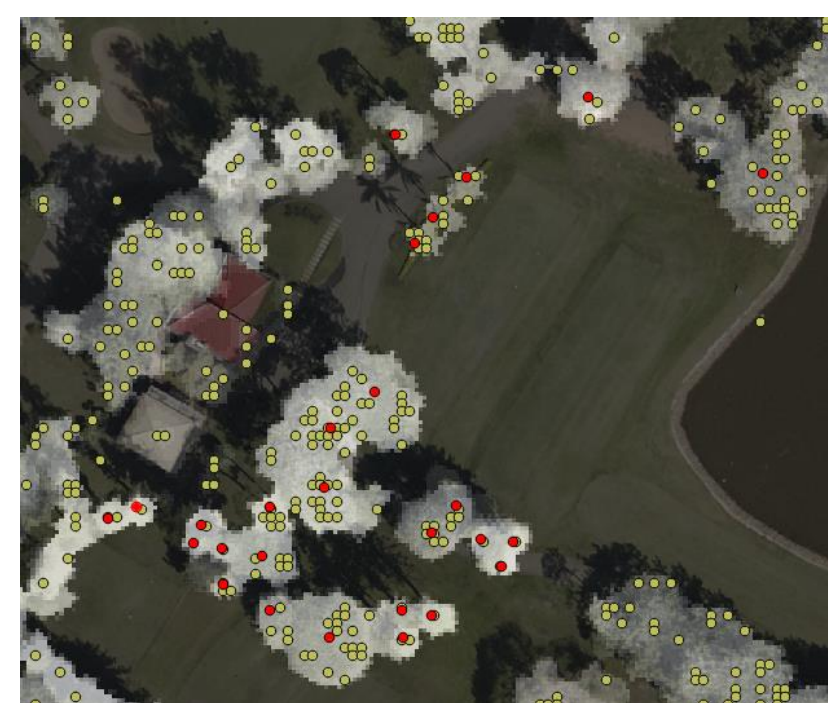

Figure 5. Extraction result of the VWF method using the pit-free CHMs

3.2.2 Results for the MMAC method: Based on mathematical morphology (MM) concepts and an adapted watershed segmentation, the MMAC method had also been implemented in this study. The best results shown as Figure 6 can be derived using pit-free CHMs (points are replaced by ten $\mathrm{cm}$ circles) in the experiments. In Figure 6, duplicated treetops appeared in the VWF results had been significantly eliminated by the MMAC method. However, there are 9 trees which cannot be found using the MMAC method, as shown in brown circle in the figure. The accuracy of the method is about $67 \%$ though it still needs to be improved. Moreover, the poor result as shown in Figure 7 is obtained when the simple subtraction CHM used for the MMAC method. It indicates that "holes" in the CHM data without smoothing will produce artifacts, which cause less amount of treetops can be found (Lin et al., 2011). More quantitative evaluations of methods will be performed in the future research.

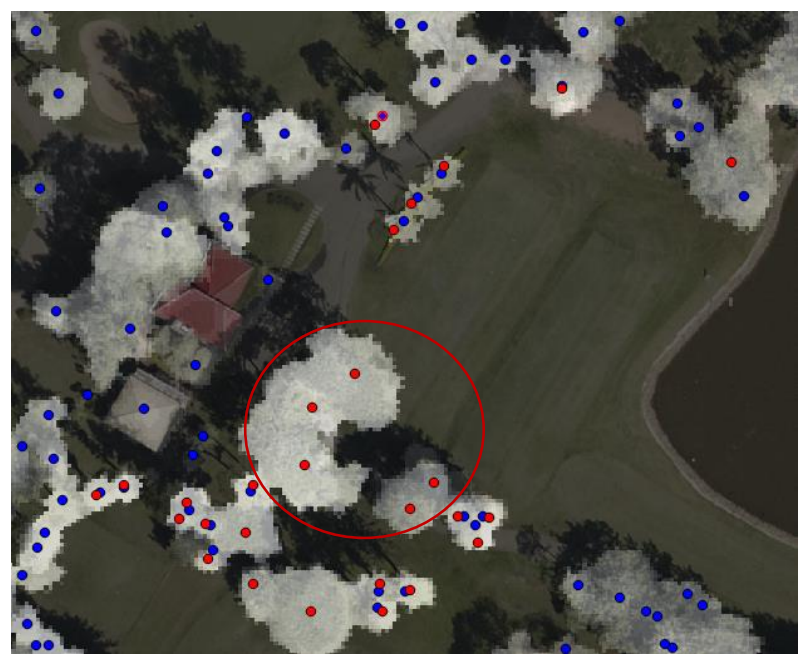

Figure 6. Extraction result by the MMAC method using the pit-free CHM (a ten cm circle as a point) 


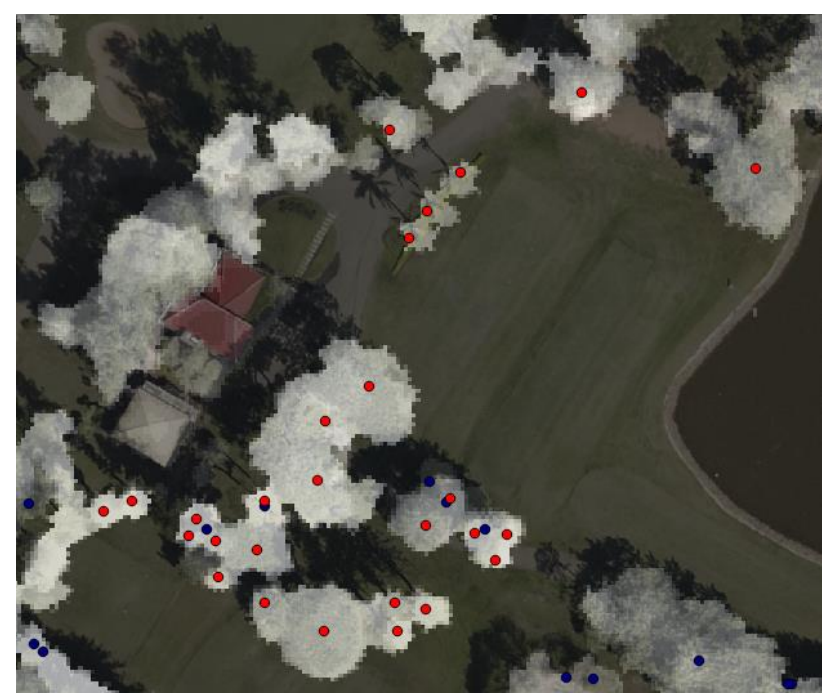

Figure 7. Extraction result by the MMAC method using the simple subtraction CHMs.

\section{CONCLUSIONS}

Two individual tree delineation algorithms namely a multilevel morphological active-contour (MMAC) and a variable window filter (VWF) had been checked and evaluated using reference data derived from the stereo measurement in the aerial image pairs in this work. The extraction results indicate that there are too many duplicated tree tops found in the VWF method, even though smoothing procedure had been considered, using pit-free CHM data. However, the duplicated treetops appeared in the VWF results had been significantly eliminated by the MMAC method. of the accuracy of the MMAC method is $67 \%$ showing that the "holes" in the CHM data without smoothing will cause more crown fragmentation and thus reducing the accuracy in the individual crown delineation. More detailed comparison and field verification will be given in the future research.

\section{ACKNOWLEDGMENTS}

The authors want to thanks for the financial support of Ministry of Science and Technology in Taiwan (Grant no. MOST 104-2119-M-159-001).

\section{REFERENCES}

Bortolot, Z. J. and Wynne, R. H., 2005. Estimating forest biomass using small footprint LiDAR data: An individual tree-based approach that incorporates training data, ISPRS J. Photogramm. Remote Sens., vol. 59, no. 6, pp. 342-360.

Dalponte, M., Bruzzone, L. and Gianelle, D, 2011. A system for the estimation of single-tree stem diameter and volume using multireturn LiDAR data, IEEE Trans. Geosci. Remote Sens., vol. 49, no. 7, pp. 2479-2490, Jul. 2011.

Isenburg, M., 2014. Rasterizing Perfect Canopy Height Models from LiDAR, Access via URL: rapidlasso.com/2014/11/04/rasterizing-perfect-canopy- height-models-from-lidar/

Khosravipour, A., Skidmore, A. K., Isenburg, M., Wang, T. J., Hussin, Y. A., 2014. Generating pit-free Canopy Height Models from Airborne LiDAR, Photogrammetric Engineering \& Remote Sensing, Vol. 80, No. 9, pp. 863872 .

Kwak, D. A., Lee, W. K., Cho, H. K., Lee, S. H., Son, Y., Kafatos, M., and Kim, S. R., 2010. Estimating stem volume and biomass of Pinus koraiensis using LiDAR data, J. Plant Res., vol. 123, no. 4, pp. 421-432.

Lin, Chinsu, Thomson, Gavin, Lo, Chien-Shun and Yang, Ming-Shein, 2011. A Multi-level Morphological Active Contour Algorithm for Delineating Tree Crowns in Mountainous Forest, Photogrammetric Engineering \& Remote Sensing, Vol. 77, No. 3, pp. 241-249.

Lo, Chien-Shun and Lin, Chinsu, 2013. GrowthCompetition-Based Stem Diameter and Volume Modeling for Tree-Level Forest Inventory Using Airborne LiDAR Data, IEEE Trans. Geosci. Remote Sens., Vol. 51, No. 4, pp. $2216-2226$.

Popescu, S. C. and R. H. Wynne, 2004. Seeing the trees in the forest: using lidar and multispectral data fusion with local filtering and variable window size for estimating tree height. Photogrammetric Engineering \& Remote Sensing, 70(5):589-604.

Popescu, S. C. 2007. Estimating biomass of individual pine trees using airborne LiDAR, Biomass Bioenergy, vol. 31, no. 9, pp. 646-655.

Silva, C.A., Hudak, A. T., Crookston, N. L., 2014. A Tutorial for " Web-LiDAR forest inventory: TreeTop application", Department of Defense Strategic Environmental Research and Development Program: Patterns and processes: monitoring and understanding plant diversity in frequently burned longleaf pine landscapes, PI: J.O’Brien; Co-PIs: R.Mitchell, A.Hudak, L.Dyer.

Wehr, A. and U. Lohr, 1999. Airborne laser scanning - an introduction and overview," ISPRS J. Photogramm. Remote Sens., International Society for Photogrammetry and Remote Sensing (ISPRS), 54, pp. 68-82. 